\title{
Isolated Double-Positive Optic Neuritis: A Case of Aquaporin-4 and Myelin Oligodendrocyte Glycoprotein Antibody Seropositivity
}

\author{
Matthew C. Mason ${ }^{1}$, Dario A. Marotta ${ }^{1,2}$, Hassan Kesserwani ${ }^{3}$ \\ 1. Department of Research, Alabama College of Osteopathic Medicine, Dothan, USA 2. Department of Neurology, \\ Division of Neuropsychology, University of Alabama, Birmingham, USA 3. Neurology, Flowers Medical Group, Dothan, \\ USA
}

Corresponding author: Matthew C. Mason, matt.mason.2022@gmail.com

\begin{abstract}
Optic neuritis (ON) causes acute vision loss with typical and atypical profiles, serological markers, imaging findings, and clinical outcomes depending on the associated underlying pathophysiology. Neuromyelitis optica (NMO) and myelin oligodendrocyte glycoprotein antibody disease (MOGAD) are the usual causes of acute severe sequential or simultaneous bilateral optic neuritis. These conditions are usually accompanied by multi-level spinal cord demyelination, and notably, they are typically positive for either NMO or Myelin oligodendrocyte glycoprotein (MOG) autoantibodies, but rarely both. We present a case of isolated sequential bilateral optic neuritis that was seropositive for both NMO and MOG antibodies.
\end{abstract}

Received 05/14/2021 Review began 05/18/2021 Review ended 05/31/2021 Published 06/02/2021

(c) Copyright 2021

Mason et al. This is an open access article distributed under the terms of the Creative Commons Attribution License CC-BY 4.0., which permits unrestricted use, distribution, and reproduction in any medium, provided the original author and source are credited.
Categories: Internal Medicine, Neurology, Ophthalmology

Keywords: double positive optic neuritis, optic neuritis, neuromyelitis optica spectrum disorder, myelin-

oligodendrocyte glycoprotein (mog), aquaporin-4 antibody, myelin-oligodendrocyte glycoprotein antibody disease

\section{Introduction}

Optic neuritis (ON) is usually a demyelinating inflammatory injury to one or both optic nerves. The typical clinical presentation of optic neuritis is unilateral acute vision loss with associated retrobulbar pain. In contrast, atypical presentations of ON have more severe vision loss that is rapidly sequential or simultaneous bilateral vision loss, steroid-resistant, and may portend a worse outcome if untreated [1]. These fundamental differences led to identifying distinct clinical conditions known as neuromyelitis optica (NMO) and myelin oligodendrocyte glycoprotein antibody disease (MOGAD).

Typical ON is a condition occurring in 1.5-5.1 cases per 100,000 with a higher incidence in females, whereas NMO and MOGAD are 0.5-1.4 per 100,000 persons [2-4]. NMO, a variant of NMO spectrum disorder (NMOSD), and MOGAD are characterized by serological positivity to aquaporin-4 (AQP-4) antibody and MOG antibody for NMO/NMOSD and MOGAD, respectively, but the frequency of double seropositivity has rarely been reported [5-7]. Here we report a 66-year-old female with steroid-responsive isolated sequential bilateral optic neuritis and seropositivity to both NMO and MOG antibodies.

\section{Case Presentation}

A 66-year-old female presented to the neurology clinic sequentially bilateral vision loss with associated retrobulbar pain on extraocular movement. The patient reports that she experienced acute onset left eye vision loss and pain that occurred without an inciting event eight weeks prior. Following initial improvement with an oral five-day tapered $48 \mathrm{mg}$ methylprednisolone pack, she developed acute onset right eye vision loss and pain. She denied any headache, numbness, weakness, vertigo, ataxia, diplopia, fatigue, urogenital dysfunction, or slurred speech, cough, shortness of breath, rashes, conjunctival erythema, and arthralgias. She also denied the Lhermitte phenomenon.

The patient reported a past medical history of hypertension and an episode of Parsonage-Turner syndrome 15 years before presentation. Her medications included amlodipine, hydrochlorothiazide, and low dose aspirin. She denied any remote or recent history of tobacco, alcohol, and illicit drug use. Family history was also unremarkable, and she denied a family history of underlying autoimmune disease.

The patient is 5 feet 6 inches, weighing 176 pounds, with a body mass index of $28.4 \mathrm{~kg} / \mathrm{m}^{2}$. Her vitals revealed a blood pressure of 142/88 mmHg, heart rate of 86 beats per minute, and oxygen saturation of $97 \%$. Physical exam revealed normal gait stance, cadence and tandem. Dilated fundoscopic exam revealed bilateral papilledema (Figure 1). 


\section{Cureus}

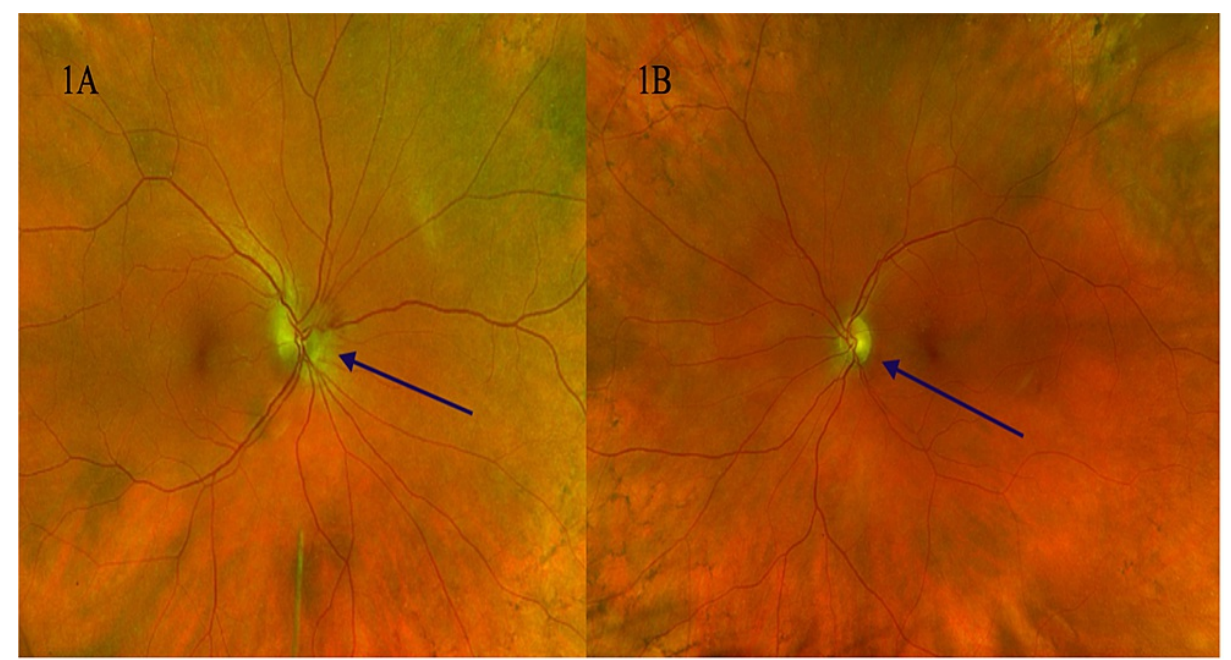

FIGURE 1: 1A: Left eye fundus, optic disc edema (blue arrow). 1B: Right eye fundus, optic disc edema (blue arrow)

Her visual acuity in the right eye was limited to counting fingers, while left eye visual acuity was 20/40. Humphrey visual field testing is shown in figure 2.

$2 \mathrm{~A}$

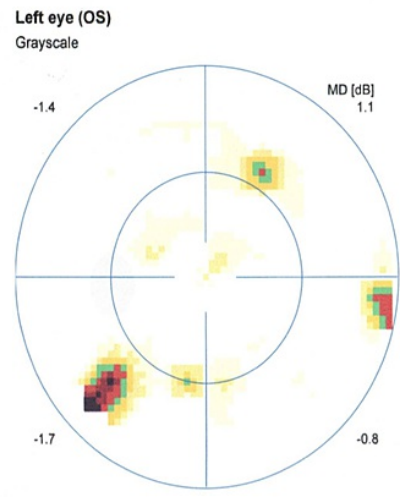

2B Right eye (OD)

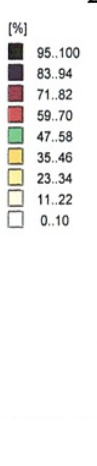

Grayscale

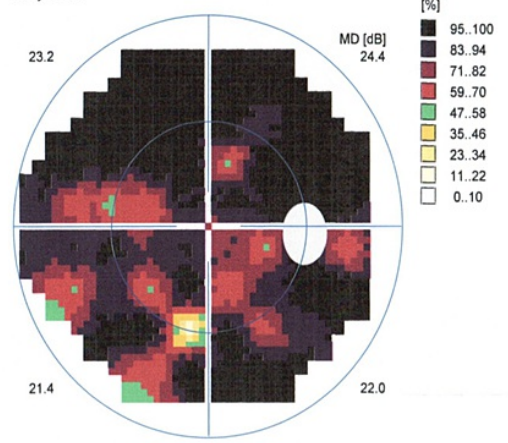

FIGURE 2: 2A: Left eye Humphrey visual field testing showing constricted visual field. 2B: Right eye Humphrey visual field testing showing near blackout of vision. Decibel (dB).

The extra-ocular motion was complete in all directions. The right eye demonstrated a Marcus-Gunn pupil. Consensual light and accommodative reflexes were intact. No ptosis was noted. Right eye visual acuity was greater than 20/200 (count fingers), left eye visual acuity was 20/40. The remaining cranial nerves were normal. Muscle strength testing in all extremities was $5 / 5$ on the medical research council grading scale. Coordination with finger-to-nose and heel-to-shin testing was normal bilaterally. Deep tendon reflexes were 2+ bilaterally in the upper and lower extremities. Sensory examination to touch-pressure, pin-prick, vibration and joint-position sense was normal in the fingers and toes. Magnetic resonance imaging (MRI) of the brain and orbits revealed enhancement of the left optic nerve with no cerebral white matter plaques (figure 3). 


\section{Cureus}

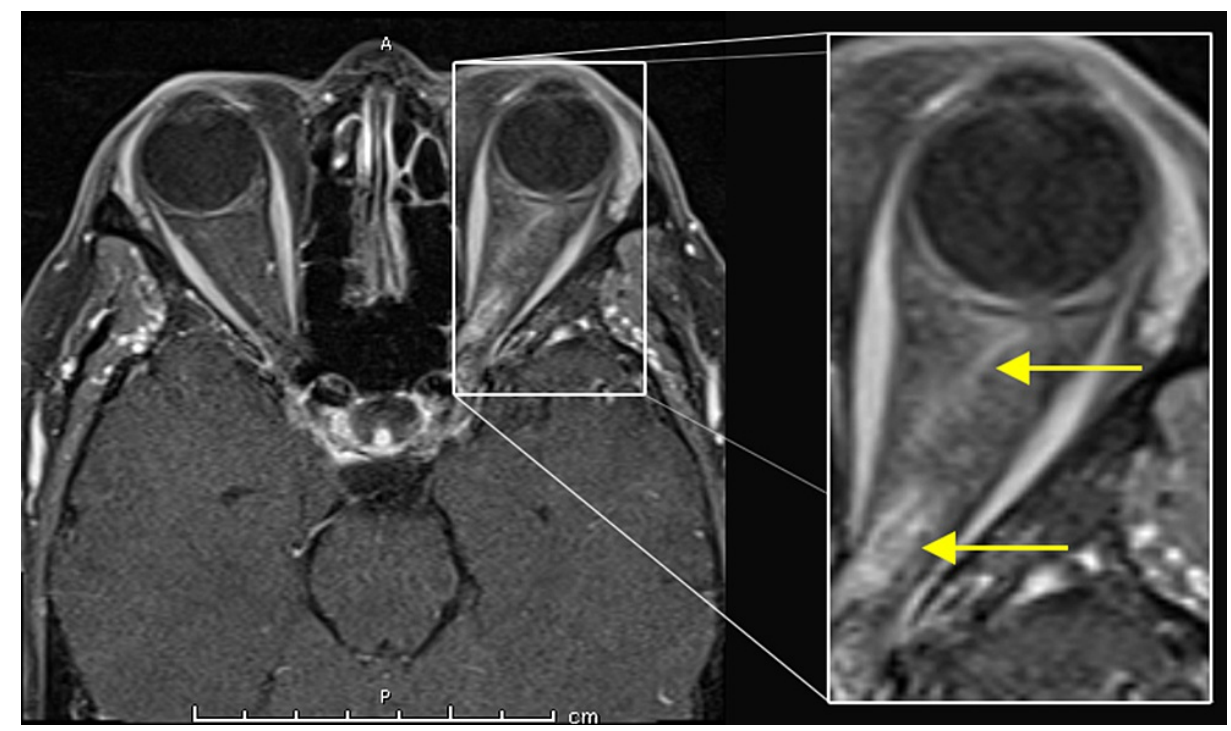

FIGURE 3: T1-weighted axial MRI gadolinium-enhanced image: enhancing and thickened optic nerve (yellow arrows)

Carotid artery duplex ultrasound of the neck revealed anterograde flow with mild, subclinical intimal thickening without significant plaque, occlusion, or stenosis. A visual evoked potential study revealed bilateral anterior visual conduction deficits with prolonged P100 latencies bilaterally, worse in the right eye. Lumbar puncture with cerebral spinal fluid and serology analyses was conducted and can be seen below in Table 1. Most notably, serum NMO and MOG antibodies were positive, while cerebral spinal fluid (CSF) markers, including CSF oligoclonal bands and intrathecal synthesis of immunoglobulins, were negative. 


\section{Cureus}

\begin{tabular}{|c|c|c|c|c|}
\hline TEST & RESULT & INTERPRETATION & REFERENCE RANGE & UNITS \\
\hline \multicolumn{5}{|l|}{ CSF } \\
\hline Color & Colorless & Normal & Colorless & N/A \\
\hline Clarity & Clear & Normal & Clear & N/A \\
\hline Total protein & 33.4 & Normal & $0.0-44.0$ & $\mathrm{mg} / \mathrm{dL}$ \\
\hline Glucose & 58 & Normal & $40-70$ & $\mathrm{mg} / \mathrm{dL}$ \\
\hline Nucleated cell & 1 & Normal & $0-5$ & cells/microliter \\
\hline $\mathrm{RBC}$ & 0 & Normal & None seen & cells/microliter \\
\hline NMO/AQP4 IgG & $<1.1$ & Normal & $<1.1$ & N/A \\
\hline Oligoclonal bands & 0 & Normal & $<4$ & N/A \\
\hline \multicolumn{5}{|l|}{ SERUM } \\
\hline NMO IgG Autoantibodies & 3.4 & Positive & $0.0-3.0$ & $\mathrm{U} / \mathrm{mL}$ \\
\hline MOG antibodies & 1:100 & Positive & $<1: 10$ & Titer \\
\hline ANA & $1: 40$ & Borderline & $<1: 40$ & Titer \\
\hline Anti-centromere antibodies & $<1: 40$ & Normal & $<1: 40$ & Titer \\
\hline RPR & Non-reactive & Normal & Non-reactive & N/A \\
\hline Anti-myeloperoxidase antibodies & $<9.0$ & Normal & $0.0-9.0$ & $\mathrm{U} / \mathrm{mL}$ \\
\hline Anti-proteinase- 3 antibodies & $<3.5$ & Normal & $0.0-3.5$ & $\mathrm{U} / \mathrm{mL}$ \\
\hline Cytoplasmic ANCA & $<1: 20$ & Normal & $<1: 20$ & Titer \\
\hline Perinuclear ANCA & $<1: 20$ & Normal & $<1: 20$ & Titer \\
\hline Atypical perinuclear ANCA & $<1: 20$ & Normal & $<1: 20$ & Tit \\
\hline
\end{tabular}

\section{TABLE 1: Results of CSF and serological analyses}

CSF = Cerebral spinal fluid, N/A = Not applicable, RBC = Red blood cells, IgG = Immunoglobulin G, ANA = Antinuclear antibody, RPR = Rapid plasma reagin, ANCA = Antineutrophil cytoplasmic antibody

Our patient's treatment course included an oral five-day 48mg tapered dose methylprednisolone pack following her initial onset of unilateral left eye vision loss, which surprisingly led to significant improvement in visual acuity. Upon developing the right eye vision loss, she was scheduled to receive the standard steroid therapy with a three-day regimen of daily one-gram intravenous methylprednisolone (IVMP). However, the patient could not tolerate IV therapy after one dose and was restarted on oral prednisone at a dose of $20 \mathrm{mg}$ twice daily. The response by the seventh day of treatment was dramatic, and she was able to read messages on her cell phone with her right eye. The plan is to taper the steroid dose by $10 \mathrm{mg}$ every month slowly.

\section{Discussion}

In recent years, atypical, clinically overlapping presentations of optic neuritis and spinal cord syndromes in combination with varying CSF and serology findings have led to the distinction of NMO, NMOSD, and MOGAD. The separation of these syndromes was further supported by discovering NMO and MOG antibodies with a relatively high degree of sensitivity and specificity. Modern serological testing for NMO have sensitivities of $75 \%$ and specificities that approach $100 \%$ [8]. It was proposed in the literature that these antibodies played independent roles in the pathophysiological development of NMO and MOGAD because the incidence of double seropositive NMO and MOG antibodies were not or rarely reported [6,9]. A review of the literature for double seropositive optic neuritis cases can be visualized below in Table 2 . 


\section{Cureus}

\section{Study}

dos Passos et al. [5]

Ishikawa et al. [10]

Woodhall et al. [11]

Matsuda et al. [12]

\section{Number of Cases}

13

1

1

2

\section{TABLE 2: Reported cases of serological double positivity to NMO and MOG antibody}

In 2015, international expert consensus outlined the diagnostic criteria for NMO, which consisted of 1) At least one core clinical characteristic (optic neuritis, transverse myelitis, area postrema syndrome, acute brainstem syndrome, symptomatic narcolepsy or acute diencephalic clinical syndrome with NMOSD-typical diencephalic MRI lesions); 2) Positive serology for AQP4-Immunoglobulin G (IgG); 3) Exclusion of alternative diagnoses [13]. In 2018, international expert consensus described the diagnostic criteria for MOGAD, which included: 1) Monophasic or relapsing acute ON, myelitis, brainstem encephalitis, or encephalitis, or any combination of these syndromes; 2) MRI or electrophysiological (visual evoked potentials in patients with isolated ON) findings compatible with CNS demyelination; 3) Seropositivity for MOG-IgG [9]. Below, we compare and contrast the findings in NMO and MOGAD (Table 3).

\begin{tabular}{|c|c|c|}
\hline Characteristic & NMO & MOGAD \\
\hline Age at onset & $\sim 40$ & $\sim 20-30$ \\
\hline $\begin{array}{l}\text { Incidence (per } \\
100,000)\end{array}$ & 0.5 & $0.2-1.4$ \\
\hline $\begin{array}{l}\text { Serological } \\
\text { antibodies }\end{array}$ & AQP-4 lgG & MOG IgG \\
\hline CSF & Oligoclonal bands in 5-10\%, mononuclear pleocytosis & $\begin{array}{l}\text { Oligoclonal bands in } 40 \% \text {, mononuclear } \\
\text { pleocytosis }\end{array}$ \\
\hline $\begin{array}{l}\text { Female:male } \\
\text { ratio }\end{array}$ & 1-2:1 & 8-9:1 \\
\hline $\begin{array}{l}\text { Clinical } \\
\text { presentation }\end{array}$ & $\begin{array}{l}\text { Optic neuritis, transverse myelitis, area postrema syndrome, } \\
\text { brainstem syndrome, narcolepsy or acute diencephalic syndrome, } \\
\text { cerebral syndrome with NMOSD-typical brain lesions }\end{array}$ & $\begin{array}{l}\text { ADEM-like (ADEM, MDEM, ADEM optic } \\
\text { neuritis, encephalitis) or opticospinal (optic } \\
\text { neuritis, myelitis) or brainstem encephalitis }\end{array}$ \\
\hline $\begin{array}{l}\text { (laterality, } \\
\text { severity, and } \\
\text { outcome) }\end{array}$ & $\begin{array}{l}\text { Bilateral more often than unilateral, often posterior optic pathway, } \\
\text { involvement of optic chiasma, long lesion, often recurrent, severe, } \\
\text { often residual deficits }\end{array}$ & $\begin{array}{l}\text { Bilateral more often than unilateral, often } \\
\text { anterior optic pathway, long lesion, often } \\
\text { recurrent, severe, good recovery }\end{array}$ \\
\hline (brain) & $\begin{array}{l}\text { Peri-ependymal lesions surrounding the ventricular system. } \\
\text { Lesions involving corticospinal tracts, or no brain lesions }\end{array}$ & $\begin{array}{l}\text { ADEM-like brain lesions, or no brain lesions, } \\
\text { long-segment spinal lesions }\end{array}$ \\
\hline $\begin{array}{l}\text { MRI findings } \\
\text { (spine) }\end{array}$ & $\begin{array}{l}\text { Long-segment lesions (>3 vertebral segments); typıcally involving } \\
\text { cervicothoracic segment; central cord predominance }\end{array}$ & $\begin{array}{l}\text { Long-segment lesions (>3 vertebral } \\
\text { segments); typically involving thoracolumbar } \\
\text { segment and conus; confined to grey matter }\end{array}$ \\
\hline Clinical course & Relapsing & Monophasic or relapsing \\
\hline
\end{tabular}

\section{TABLE 3: Comparison of NMO and MOGAD characteristics[14]}

ADEM = Acute disseminated encephalomyelitis, MDEM = Multiphasic disseminated encephalomyelitis, CSF- Cerebrospinal fluid, MOG- Myelin oligodendrocyte glycoprotein, IgG- Immunoglobulin G, NMO- Neuromyelitis optica, MOGAD- myelin oligodendrocyte glycoprotein antibody disease, AQP-4- Aquaporin-4

We present a case of double seropositive sequential bilateral ON. This presentation is sporadic due to the double seropositivity itself, but even more so because of the late age of onset, isolated ON without clinical 
signs of acute myelitis and area postrema syndrome, and lack of characteristic MRI findings. Additionally, our case can reasonably be diagnosed using both NMO and MOGAD expert consensus diagnostic criteria despite these conditions having different antigen targets and unique underlying pathophysiology. Intravenous steroid therapy has been the gold standard treatment for ON with potential add-on therapies with plasma exchange therapy, T-cell modulators, and B-cell modulators for ON cases with steroid resistance or recurrence $[15,16]$. In a retrospective review of 70 patients with MOGAD on maintenance therapy, intravenous immunoglobulin was associated with the lowest median annualized relapse rate, followed by azathioprine, rituximab and mycophenolate mofetil [17]. Our patient obtained notable improvement in visual acuity with oral steroid therapy and continued oral therapy following the onset of bilateral $\mathrm{ON}$ as she could not tolerate IV therapy due to cumbersome side effects.

Upon reviewing the literature, there is an apparent paucity of patients with double seropositivity to NMO and MOGAD antibodies. The clinical outcomes for NMO and MOGAD are well established. A diagnosis of NMO with positivity to AQP-4 antibody portends a much higher relapse rate with an unfavourable outcome compared to NMO with negative AQP-4 antibody or isolated MOGAD. However, the prognosis of double positivity $\mathrm{ON}$ is unknown. While our patient responded quite well to steroid therapy, future studies on double seropositive $\mathrm{ON}$ would provide clinical benefit and clarity to a rather unique condition.

\section{Conclusions}

Bilateral optic neuritis is an interesting condition that has recently been increasingly recognized and better characterized. The discovery of the immune markers AQP-4 and MOG antibodies has revolutionized the approach to these patients. Treatment algorithms are being refined and defined. It is common practice to refer to optic neuritis, especially bilateral optic neuritis, as NMO-positive, MOG-positive, double-positive or double-negative. The arsenal of therapeutics includes IV steroids, intravenous immunoglobulin, plasma exchange and surprisingly oral steroids, as in our case. Our case highlights the unexpected benefit of oral steroids and shows that testing for these autoimmune markers is a prerequisite in the work-up of patients with optic neuritis. As the data and research continue to unfold, we will be better positioned to develop treatment algorithms.

\section{Additional Information \\ Disclosures}

Human subjects: Consent was obtained or waived by all participants in this study. Conflicts of interest: In compliance with the ICMJE uniform disclosure form, all authors declare the following: Payment/services info: All authors have declared that no financial support was received from any organization for the submitted work. Financial relationships: All authors have declared that they have no financial relationships at present or within the previous three years with any organizations that might have an interest in the submitted work. Other relationships: All authors have declared that there are no other relationships or activities that could appear to have influenced the submitted work.

\section{References}

1. Papais-Alvarenga RM, Carellos SC, Alvarenga MP, Holander C, Bichara RP, Thuler LC: Clinical course of optic neuritis in patients with relapsing neuromyelitis optica. Arch Ophthalmol. 2008, 126:12-16. 10.1001/archophthalmol.2007.26

2. Horton L, Bennett JL: Acute management of optic neuritis: an evolving paradigm . J Neuroophthalmol. 2018, 38:358-367. 10.1097/wno.0000000000000700

3. de Mol CL, Wong Y, van Pelt ED, et al.: The clinical spectrum and incidence of anti-MOG-associated acquired demyelinating syndromes in children and adults. Mult Scler. 2020, 26:806-814. $10.1177 / 1352458519845112$

4. Sepúlveda M, Aldea M, Escudero D, et al.: Epidemiology of NMOSD in catalonia: influence of the new 2015 criteria in incidence and prevalence estimates. Mult Scler. 2018, 24:1843-1851. 10.1177/1352458517735191

5. dos Passos GR, Oliveira LM, da Costa BK, Apostolos-Pereira SL, Callegaro D, Fujihara K, Sato DK: MOG-IgGassociated optic neuritis, encephalitis, and myelitis: lessons learned from neuromyelitis optica spectrum disorder. Front Neurol. 2018, 9:217. 10.3389/fneur.2018.00217

6. Sato DK, Callegaro D, Lana-Peixoto MA, et al.: Distinction between MOG antibody-positive and AQP4 antibody-positive NMO spectrum disorders. Neurology. 2014, 82:474-481. 10.1212/wnl.0000000000000101

7. Dubey D, Pittock SJ, Krecke KN, et al.: Clinical, radiologic, and prognostic features of myelitis associated with myelin oligodendrocyte glycoprotein autoantibody. JAMA Neurol. 2019, 76:301. 10.1001/jamaneurol.2018.4053

8. Höftberger R, Sabater L, Marignier R, et al.: An optimized immunohistochemistry technique improves NMOIgG detection: study comparison with cell-based assays. PLoS One. 2013, 8:e79083. 10.1371/journal.pone.0079083

9. Jarius S, Paul F, Aktas O, et al.: MOG encephalomyelitis: international recommendations on diagnosis and antibody testing. J Neuroinflammation. 2018, 15:134. 10.1186/s12974-018-1144-2

10. Ishikawa H, Kezuka T, Shikishima K, et al.: Epidemiologic and clinical characteristics of optic neuritis in japan. Ophthalmology. 2019, 126:1385-1398. 10.1016/j.ophtha.2019.04.042

11. Woodhall M, Coban A, Waters P, et al.: Glycine receptor and myelin oligodendrocyte glycoprotein antibodies in Turkish patients with neuromyelitis optica. J Neurol Sci. 2013, 335:221-223. 10.1016/j.jns.2013.08.034

12. Matsuda R, Kezuka T, Umazume A, Okunuki Y, Goto H, Tanaka K: Clinical profile of anti-myelin 


\section{Cureus}

oligodendrocyte glycoprotein antibody seropositive cases of optic neuritis. Neuroophthalmology. 2015, 39:213-219. 10.3109/01658107.2015.1072726

13. Wingerchuk DM, Banwell B, Bennett JL, et al.: International consensus diagnostic criteria for neuromyelitis optica spectrum disorders. Neurology. 2015, 85:177-189. 10.1212/wnl.0000000000001729

14. Hegen H, Reindl M: Recent developments in MOG-IgG associated neurological disorders . Ther Adv Neurol Disord. 2020, 13: 10.1177/1756286420945135

15. Beck RW, Cleary PA, Anderson MM, et al.: A randomized, controlled trial of corticosteroids in the treatment of acute optic neuritis. N Engl J Med. 1992, 326:581-588. 10.1056/nejm199202273260901

16. Watanabe S, Nakashima I, Misu T, Miyazawa I, Shiga Y, Fujihara K, Itoyama Y: Therapeutic efficacy of plasma exchange in NMO-IgG-positive patients with neuromyelitis optica. Mult Scler. 2007, 13:128-132. $10.1177 / 1352458506071174$

17. Chen JJ, Flanagan EP, Bhatti MT, et al.: Steroid-sparing maintenance immunotherapy for MOG-IgG associated disorder. Neurology. 2020, 95:111-120. 10.1212/WNL.0000000000009758 